\title{
EXTRAÇÃO DE PIMENTA MALAGUETA (Capsicum frutescens L.) A BAIXA PRESSÃO ASSISTIDA POR ULTRASSOM
}

\author{
A. L. B. DIAS ${ }^{1}$, P. SANTOS ${ }^{1}$, A. C. AGUIAR ${ }^{1}$, G. F. BARBERO² e J. MARTÍNEZ ${ }^{1}$ \\ ${ }^{1}$ Departamento de Engenharia de Alimentos, Faculdade de Engenharia de Alimentos, \\ Universidade Estadual de Campinas (UNICAMP), Campinas, SP, Brasil \\ E-mail para contato: julian@ fea.unicamp.br \\ 2Departamento de Química Analítica, Faculdade de Ciências, Universidade de Cadiz, \\ Campus Excelencia Internacional Agroalimentario, Puerto Real, Cádiz, Espanha
}

\begin{abstract}
RESUMO - A técnica de ultrassom baseia-se na formação de ondas ultrassônicas de alta frequência, capazes de provocar cavitação e causar ruptura na parede celular da matriz vegetal. Isto favorece a penetração do solvente, a transferência de massa e, consequentemente, o aumento do rendimento de extrato. Neste contexto, o objetivo principal do presente trabalho foi obter extratos de pimenta malagueta através da utilização do processo de extração a baixa pressão assistido por ultrassom. A matéria prima utilizada foi a pimenta malagueta desidratada e triturada. Para estudar a influência das variáveis do processo na extração, foram realizados experimentos com e sem ultrassom, nas potências e temperaturas variando de 200 a $600 \mathrm{~W}$ e 40 a $60{ }^{\circ} \mathrm{C}$, respectivamente. Em todos os ensaios os parâmetros razão entre matéria prima e solvente e tempo de extração (20 minutos) foram mantidos constantes. A partir dos resultados obtidos pôde-se observar um aumento no rendimento global, na presença de ondas ultrassônicas.
\end{abstract}

\section{INTRODUÇÃO}

As pimentas vermelhas (Capsicum sp.) são vegetais ricos em capsaicinoides, substâncias responsáveis pela pungência dos frutos, tendo a capsaicina como sua maior representante (DAMODARAN, PARKIN e FENNEMA, 2008). Atualmente a capsaicina é empregada no desenvolvimento de novos fármacos por possuir várias propriedades benéficas, tais como poder antioxidante, antimicrobiano, anti-inflamatório e antitumoral, além de contribuir com o controle de diabetes e alívio de dores (REYESESCOGIDO, GONZALEZ-MONDRAGON e VAZQUEZ-TZOMPANTZI, 2011). Tendo em vista o poder benéfico da capsaicina, existe um grande interesse no desenvolvimento de novas tecnologias para a obtenção de extratos concentrados dessa substância.

A extração de compostos ativos de matérias-primas de origem vegetal é um campo promissor na indústria de alimentos. Por outro lado, é uma tarefa complexa, pois na maioria das vezes esses compostos são substâncias termolábeis e susceptíveis a oxidação. Além disso, restrições legais estão propondo a eliminação do uso geral de solventes orgânicos em plantas industriais de extração. Portanto, há um interesse considerável em substituir processos como destilação a vapor e extrações com solventes orgânicos, tradicionalmente utilizados para obter estes compostos ativos (MARTINEZ, 2008). 
A técnica de ultrassom baseia-se na formação de ondas ultrassônicas de alta frequência capazes de provocar cavitação devido aos ciclos de expansão e contração sofridos pela matéria. Tais ciclos causam ruptura na parede celular da matriz vegetal, favorecendo a penetração do solvente e a transferência de massa, consequentemente, o aumento do rendimento de extrato (TOMA et al., 2001). Além disso, a utilização da técnica de ultrassom resulta em um novo arranjo na microestrutura da matéria. (UQUICHE, VALLE e ORTIZ, 2004).

Devido ao exposto, o objetivo principal do trabalho foi obter extratos de pimenta malagueta, visando ao maior rendimento global, através de diferentes técnicas de extração a baixa pressão. Adicionalmente, pretendeu-se avaliar as variáveis do processo da técnica de extração assistida por ultrassom, temperatura e potência, bem como comparar os teores de capsaicinoides obtidos por tal técnica com a metodologia convencional de obtenção desses compostos.

\section{MATERIAL E MÉTODOS}

O presente trabalho foi realizado no Laboratório de Alta Pressão em Engenharia de Alimentos (LAPEA), Faculdade de Engenharia de Alimentos (DEA/UNICAMP), em Campinas-SP. A matéria-prima utilizada foi a pimenta da variedade malagueta (Capsicum frutescens L.) adquirida na Central de Abastecimento de Campinas S.A. (CEASA, Campinas-SP). Os frutos foram selecionados quanto à sua integridade física e, posteriormente, higienizados através da imersão em uma solução de $100 \mathrm{ml} / \mathrm{L} \mathrm{de}$ hipoclorito de sódio durante 20 minutos. Após esse período, os frutos foram enxaguados com água corrente para a retirada do excesso da solução higienizadora e finalmente armazenados em freezer $\left(-18{ }^{\circ} \mathrm{C}\right)$ para futura utilização.

A matéria-prima foi submetida ao processo de secagem em estufa com circulação de ar forçada por $70 \pm 2{ }^{\circ} \mathrm{C}$ durante 20 horas, conforme a metodologia utilizada por Aguiar et al. (2013). Após a secagem, as amostras foram moídas em moinho de facas (Marconi, modelo MA 340, Piracicaba-SP) com o objetivo de homogeneizá-las e diminuir a resistência à transferência de massa durante as etapas de extração. A classificação do material moído foi realizada conforme o tamanho de partículas em um sistema vibratório (Bertel, modelo 1868, Caieiras-SP) com peneiras de abertura de 16 a 80 mesh (série Tyler, Wheeling, EUA). As massas retidas sobre cada peneira foram pesadas em balança analítica (Radwag, modelo AS 220/C/2, São Paulo-SP). O diâmetro médio das partículas foi determinado através do modelo proposto por A.S.A.E. (1998) com um valor de aproximadamente $0,94 \pm 0,03 \mathrm{~mm}$.

A extração a baixa pressão assistida por ultrassom foi realizada em um sistema composto por um célula encamisada de vidro de $100 \mathrm{ml}$, banho de aquecimento, e um sistema de ultrassom composto por um transdutor, controlador e sonda ultrassônica com diâmetro (D) de aproximadamente $1 \mathrm{~cm}$, conforme ilustra a Figura 1. A altura da sonda ultrassônica em relação ao becker encamisado (h) foi mantida constate em $5 \mathrm{~cm}$ para todos os experimentos com ultrassom. As extrações com e sem ultrassom foram realizadas com aproximadamente $3 \mathrm{~g}$ de matéria-prima e $60 \mathrm{ml}$ solvente (etanol (Êxodo Científica/Hortolânida-SP, Brasil)), com uma razão mássica de 1:20. Além disso, o tempo de extração foi mantido constante em 20 minutos. As variáveis estudadas foram potência ultrassônica e temperatura de processo tendo como resposta o rendimento global de extrato, calculado pela relação entre massa de extrato (g) por massa de matéria-prima alimentada em cada experimento $(\mathrm{g})$. 


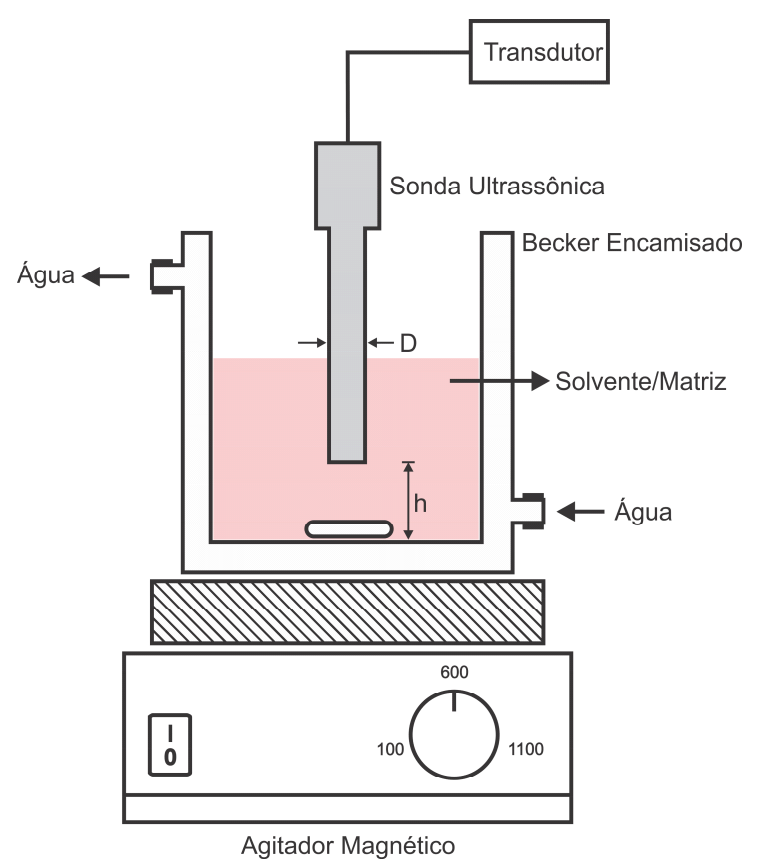

Figura 1 - Sistema de extração a baixa pressão assistido por ultrassom; D - Diâmetro da sonda ultrassônica $(\mathrm{cm}) ; \mathrm{h}$ - Posição da sonda ultrassônica $(\mathrm{cm})$.

Para comparar a extração assistida por ultrassom com uma extração convencional usando solvente orgânico, as amostras foram submetidas à técnica convencional de extração Soxhlet. Aproximadamente $5 \mathrm{~g}$ de matriz vegetal seca e moída foram pesadas em um cartucho de papel filtro, sendo o mesmo inserido em um extrator tipo Soxhlet. A razão mássica entre solvente e matéria-prima foi de 1:20 e o tempo de extração foi de seis horas. Foram utilizados quatro solventes diferentes: hexano P.A. (Êxodo Científica/Hortolânida-SP, Brazil), álcool etílico P.A. (Êxodo Científica), éter etílico P.A. (Êxodo Científica) e acetato de etila P.A. (Êxodo Científica). Ao final de seis horas o extrato foi recolhido e o solvente residual evaporado sob vácuo em um evaporador rotativo (Heildoph Instruments modelo Laborota 4001, Viertrieb).

As condições de processo extremas, $600 \mathrm{~W}$ a $60{ }^{\circ} \mathrm{C}$ e $200 \mathrm{~W}$ a $40{ }^{\circ} \mathrm{C}$ respectivamente, foram comparadas com a técnica convencional de obtenção de capsaicinoides (BARBERO et al., 2008). Aproximadamente $1 \mathrm{~g}$ dos extratos obtidos foi pesado, diluído em metanol (Êxodo Científica), ajustado a um volume de $25 \mathrm{~mL}$, e filtrado com o auxílio de filtrosmillex PVDF $0.22 \mu \mathrm{m}$ (Millipore). Por fim, as amostras foram submetidas a um banho ultrassônico (135 W/25 kHZ - Unique ultracleaner 1450) durante 20 minutos com a finalidade de homogeneizar os extratos. As amostras da matriz vegetal desidratada foram submetidas ao mesmo processo em banho ultrassônico, conforme a metodologia de Barbero et al., (2008). Uma alíquota de 1,5 mL da solução metanol/extrato foi acondicionada em um frasco âmbar para análise cromatográfica (HPLC).

Todos os ensaios foram realizados em duplicata e os resultados obtidos para rendimento global foram submetidos à análise de variância (ANOVA) e teste de Tukey, a 5\% de significância, utilizando o software Statistica 6.0. 


\section{RESULTADOS E DISCUSSÃO}

A Tabela 1 apresenta os rendimentos globais obtidos para todos os experimentos realizados na obtenção de extratos de pimenta malagueta.

Tabela 1 - Rendimentos globais obtidos para diferentes técnicas de extração em diferentes condições de processo.

\begin{tabular}{|c|c|c|c|}
\hline Método & Solvente & & Rendimento (\%) \\
\hline \multirow{5}{*}{ Soxhlet } & Hexano & & $7,98 \pm 0,27^{\text {cde }}$ \\
\hline & Álcool Etílico & & $14,0 \pm 0,34^{\mathrm{a}}$ \\
\hline & Acetato de Etila & & $9,20 \pm 0,20^{\mathrm{bc}}$ \\
\hline & Diclorometano & & $9,30 \pm 0,30^{\mathrm{b}}$ \\
\hline & Éter Etílico & & $8,60 \pm 0,40^{\mathrm{bcd}}$ \\
\hline Extração US & Temperatura $\left({ }^{\circ} \mathrm{C}\right)$ & Potência (W) & \\
\hline \multirow{3}{*}{ Sem US } & 40 & - & $5,04 \pm 0,42^{\mathrm{h}}$ \\
\hline & 50 & - & $5,93 \pm 0,17^{\text {gh }}$ \\
\hline & 60 & - & $6,71 \pm 0,07^{\mathrm{fg}}$ \\
\hline \multirow{9}{*}{ Com US } & \multirow{3}{*}{40} & 200 & $6,19 \pm 0,24^{\mathrm{gh}}$ \\
\hline & & 440 & $7,09 \pm 0,33^{\text {efg }}$ \\
\hline & & 600 & $7,08 \pm 0,22^{\text {efg }}$ \\
\hline & \multirow{3}{*}{50} & 200 & $6,53 \pm 0,06^{\mathrm{fg}}$ \\
\hline & & 440 & $7,67 \pm 0,26^{\mathrm{def}}$ \\
\hline & & 600 & $7,51 \pm 0,13^{\text {def }}$ \\
\hline & \multirow{3}{*}{60} & 200 & $7,48 \pm 0,01^{\text {def }}$ \\
\hline & & 440 & $8,19 \pm 0,15^{\text {bcde }}$ \\
\hline & & 600 & $8,37 \pm 0,40^{\mathrm{bcd}}$ \\
\hline
\end{tabular}

US - Ultrassom; Médias seguidas das mesmas letras não diferem entre si ao nível de significância de 5\% pelo teste de Tukey.

Pode-se verificar na Tabela 1 que os rendimentos globais obtidos pelo método de Soxhlet, exceto utilizando hexano e éter etílico como solvente, diferenciaram-se dos valores de rendimento global obtidos nas extrações a baixa pressão com e sem ultrassom utilizando etanol como solvente, em todas as condições estudadas. Tal comportamento é explicado devido às condições de temperatura, reciclo do solvente e interações solvente/soluto do método de Soxhlet, que contribuem para a maior solubilização dos solutos da matéria-prima (MARKOM et al., 2007; MAZZUTTI et al., 2012). O baixo rendimento obtido para os experimentos com hexano e éter etílico pode ser explicado devido à polaridade do solvente, uma vez que o hexano tem polaridade menor que a do etanol, que por sua vez teve o maior rendimento entre os solventes testados.

As extrações a baixa pressão sem ultrassom obtiverem um rendimento global de 5,04 a 6,71 gramas de extrato por grama de matriz vegetal, ou seja, observa-se que tal variação ocorreu devido ao aumento da temperatura de processo, que possivelmente é a responsável por afetar diferentes propriedades do sistema, como viscosidade, difusividade, solubilidade, pressão de vapor e tensão superficial (BOONKIRD, PHISALAPHONG e PHISALAPHONG, 2008). Quando aplicadas ondas ultrassônicas 
a tal processo o rendimento na menor condição $\left(40{ }^{\circ} \mathrm{C}\right.$ e $\left.200 \mathrm{~W}\right)$ foi de aproximadamente $6,2 \%$, valor este próximo ao rendimento obtido a $60{ }^{\circ} \mathrm{C}$ sem ultrassom. Por sua vez, quando aumentadas a temperatura e a potência, o rendimento global atingiu um valor de aproximadamente $8,37 \%$, um aumento de $80 \%$ em relação à extração sem ultrassom na mesma condição $\left(60{ }^{\circ} \mathrm{C}\right.$ e $\left.600 \mathrm{~W}\right)$.

A Tabela 2 apresenta a análise de variância dos efeitos da temperatura e potência ultrassônica sobre o rendimento.

Tabela 2 - Análise de variância (ANOVA) dos efeitos da temperatura de processo e a potência ultrassônica aplicada para o rendimento global de extrato de pimenta malagueta.

\begin{tabular}{ccccccc}
\hline & Fator & GL & SQ & MQ & $F_{\text {calc }}$ & $p$-valor \\
\hline 1 & Temperatura & 2 & 3,3882 & 1,6941 & 55,07 & 0,0001 \\
2 & Potência & 2 & 4,6016 & 2,3008 & 74,79 & 0,0001 \\
& $1 * 2$ & 4 & 0,1049 & 0,0262 & 0,85 & 0,527 \\
& Erro & 9 & 0,2769 & 0,0308 & & \\
& Total & 17 & 8,3715 & & & \\
& &
\end{tabular}

onde: $\mathrm{SQ}$ - soma dos quadrados; GL - graus de liberdade; $\mathrm{MQ}$ - quadrado médio. $\mathrm{F}_{\text {tab }}(8 ; 9 ; 0,05)=3,23$.

Pode-se verificar na Tabela 2 e na Figura 2, que mostra as isotermas de rendimento global, que os efeitos da temperatura e potência foram significativos ao nível de 5\%, ou seja, houve um aumento no rendimento quando os níveis de tais variáveis foram aumentados. Por outro lado, pôde-se verificar que a interação entre os dois fatores não foi significativa ao nível de 5\%. Observa-se tal comportamento na Figura 2, onde não ocorreu o cruzamento das isotermas de rendimentos global.

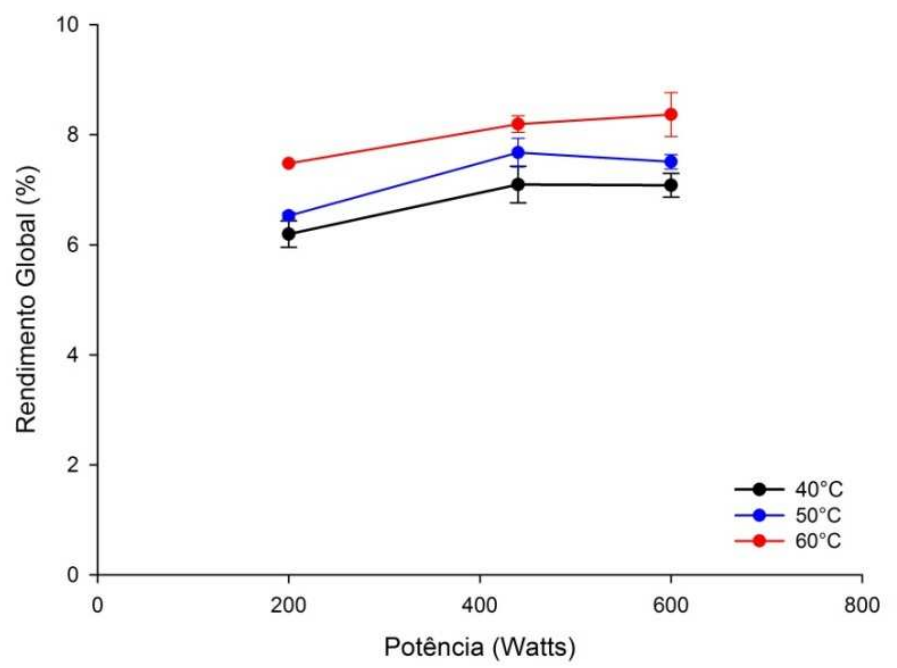

Figura 2 - Efeito da potência de ultrassom em função da temperatura de processo para o rendimento global de extrato de pimenta malagueta.

A potência ultrassônica foi responsável pelo maior efeito sobre o rendimento global $\left(\mathrm{F}_{\mathrm{cal}}=74,79\right)$. Tal comportamento pode ser explicado pelas cavitações sobre o 
sistema solvente/matriz vegetal. Tais cavitações são geradas a partir da implosão de bolhas formadas pelas ondas ultrassônicas, e caso essas bolhas se rompam próximas à matriz vegetal poderá ocorrer a quebra da parede celular, aumentando assim a área de contato entre o solvente e a matriz vegetal, e consequentemente intensificando a transferência de massa do processo (CASTRO e CAPOTE, 2007; ESCLAPEZ et al., 2011). Além disso, foi observado que, quanto maior o nível da potência ultrassônica, maior foi o rendimento global, ou seja, maior potência significa maior energia por unidade de tempo, o que possivelmente intensificou o efeito de cavitação.

A Tabela 3 apresenta o teor de capsaicinoides obtidos através da técnica convencional e os teores obtidos na maior e menor condição de processo de extração assistida por ultrassom.

Tabela 3 - Comparação entre a técnica convencional de extração de capsaicinoides e a técnica de ultrassom em diferentes condições de processo.

\begin{tabular}{cccc}
\hline Capsaicinoides & $\begin{array}{c}\text { Técnica } \\
\text { Convencional }\end{array}$ & $600 \mathrm{~W} / 60^{\circ} \mathrm{C}$ & $200 \mathrm{~W} / 40^{\circ} \mathrm{C}$ \\
\hline Capsaicina (C) & $1.89 \pm 0.06^{\mathrm{b}}$ & $3,32 \pm 0,09 \mathrm{a}$ & $3,16 \pm 0,26 \mathrm{a}$ \\
Dihidrocapsaicina (DHC) & $0,84 \pm 0,04^{\mathrm{b}}$ & $1,44 \pm 0,05^{\mathrm{a}}$ & $1,36 \pm 0,12^{\mathrm{a}}$ \\
Nordihidrocapsaicina (n-DHC) & $0,19 \pm 0,01^{\mathrm{b}}$ & $0,33 \pm 0,01^{\mathrm{a}}$ & $0,31 \pm 0,02^{\mathrm{a}}$ \\
Homodihidrocapsaicina (h-DHC) & $0,09 \pm 0,01^{\mathrm{b}}$ & $0,15 \pm 0,01^{\mathrm{a}}$ & $0,15 \pm 0,01^{\mathrm{a}}$ \\
Homocapsaicina (h-C) $_{\text {Total }}$ & $0,14 \pm 0,01^{\mathrm{b}}$ & $0,25 \pm 0,01^{\mathrm{a}}$ & $0,24 \pm 0,02^{\mathrm{a}}$ \\
\hline Tom & $3,14 \pm 0,11^{\mathrm{b}}$ & $5,50 \pm 0,16^{\mathrm{a}}$ & $5,22 \pm 0,43^{\mathrm{a}}$ \\
\hline
\end{tabular}

Médias seguidas das mesmas letras na mesma linha não diferem entre si ao nível de significância de 5\% pelo teste de Tukey.

Os resultados obtidos na análise cromatográfica demostraram que a técnica de ultrassom a baixa pressão foi eficaz na extração dos capsaicinoides da pimenta malagueta, em comparação com a técnica convencional de extração. $O$ total de capsaicinoides para a técnica convencional foi de 3,14 mg de capsaicinoides por grama de matéria-prima, valor próximo ao obtido por Aguiar et al. (2013), enquanto para a técnica aplicada de ultrassom na maior condição de processo obteve um média de aproximadamente $5,5 \mathrm{mg} / \mathrm{g}$, um aumento de aproximadamente $75 \%$. Tal comportamento pode ser explicado devido as diferentes condições entre tais técnicas. A técnica convencional utiliza metanol e um banho ultrassônico, enquanto nas extrações com ultrassom foi utilizado um solvente menos polar, etanol, que aumenta a solubilização dos capsaicinoides, e uma sonda ultrassônica (probe) que fornece uma melhor distribuição das ondas ultrassônicas no sistema solvente-matriz vegetal. Tais fatores acarretaram em um efeito positivo sobre o rendimento global e o teor de capsaicinoides.

\section{CONCLUSÃO}

As extrações de pimenta malagueta com etanol como solvente obtiveram maiores rendimentos em comparação a outros solventes testados. A técnica de extração assistida 
por ultrassom obteve maiores rendimentos globais que as extrações sem ultrassom, com um aumento de 75\%. O maior rendimento global obtido para tal técnica foi de $8,37 \%$, sendo que foi possível obter um extrato mais concentrado de capsaicinoides do que a técnica convencional de extração de tais compostos. De maneira geral, a técnica de ultrassom se mostrou eficaz na obtenção de extratos de pimenta malagueta.

\section{AGRADECIMENTOS}

Os autores gostariam de agradecer a CAPES, CNPq (147260/2013-2 e 142373/2013-3) e FAPESP (2013/02203-6) pelo suporte financeiro.

\section{REFERÊNCIAS}

A.S.A.E. Method of Determining and Expressing Fineness of Feed Materials by Sieving. American Society of Agricultural Engineers Standards. S319.3: 447-550 p. 1998.

AGUIAR, A. C., SALES L. P., COUTINHO, J.P., BARBERO, G.F., GODOY, H.T., MARTINEZ, J., Supercritical carbon dioxide extraction of Capsicum peppers: Global yield and capsaicinoid content, The Journal of Supercritical Fluids, 81, 2013, 210-216.

BARBERO, G. F. et al. Ultrasound-assisted extraction of capsaicinoids from peppers. Talanta, v. 75, n. 5, p. 1332-1337, 2008.

BOONKIRD, S.; PHISALAPHONG, C.; PHISALAPHONG, M. Ultrasound-assisted extraction of capsaicinoids from Capsicum frutescens on a lab- and pilot-plant scale. Ultrasonics Sonochemistry, v. 15, n. 6, p. 1075-1079, 2008.

CASTRO, L.; CAPOTE, F. P. Analytical Applications of Ultrasound. Elsevier, 2007. 1-298.

DAMODARAN, S.; PARKIN, K. L.; FENNEMA, O. R. Fennema's food chemistry. Boca Raton :: CRC Press/Taylor \& Francis 2008.

DE AGUIAR, A. C. et al. Supercritical carbon dioxide extraction of Capsicum peppers: Global yield and capsaicinoid content. The Journal of Supercritical Fluids, v. 81, n. 0, p. 210-216, 2013.

ESCLAPEZ, M. D. et al. Ultrasound-Assisted Extraction of Natural Products. Food Engineering Reviews, v. 3, n. 2, p. 108-120, 2011.

MARKOM, M. et al. Extraction of hydrolysable tannins from Phyllanthus niruri Linn.: Effects of solvents and extraction methods. Separation and Purification Technology, v. 52, n. 3, p. 487-496, 2007. 
MARTINEZ, J. L. Supercritical fluid extraction of nutraceuticals and bioactive compounds. . Boca Raton-FL: CRC Press, 2008.

MAZZUTTI, S. et al. Supercritical fluid extraction of Agaricus brasiliensis: Antioxidant and antimicrobial activities. The Journal of Supercritical Fluids, v. 70, n. 0, p. 48-56, 2012.

REYES-ESCOGIDO, M.; GONZALEZ-MONDRAGON， E. G.; VAZQUEZTZOMPANTZI, E. Chemical and Pharmacological Aspects of Capsaicin. Molecules, v. 16, n. 2, p. 1253-1270, 2011.

TOMA, M. et al. Investigation of the effects of ultrasound on vegetal tissues during solvent extraction. Ultrasonics Sonochemistry, v. 8, n. 2, p. 137-142, 2001.

UQUICHE, E.; VALLE, J.; ORTIZ, J. Supercritical carbon dioxide extraction of red pepper (Capsicum annuum L.) oleoresin. Journal of food engineering., v. 65, n. 1, p. 55-66, 2004. 